\title{
APUNTES PARA UNA VISIÓN EXTERNALISTA DE LA FILOSOFÍA DE DESCARTES
}

Elia Nathan

INSTITUTO DE INVESTIGACIONES FLOSÓFICAS Universidad Nacional autónoma de México

En este ensayo intentaré abogar por una visión más externalista de la historia de la filosofía que la que típicamente aparece en los textos de historia de la filosofía. Por externalismo entenderé aquí la tesis muy general de que el desarrollo de la filosofía no está tan sólo determinado por problemas internos a la filosofía sino también por factores extrafilosóficos que, aunque tienen fundamentalmente un carácter cultural, también responden a un orden socio-económico. $\mathbf{E l}$ argumento que daré en favor de esta concepción no será a priori, sino que consistirá en mostrar, a través del estudio de un caso concreto, qué ventajas reales puede aportar una visión más externalista que la usual. El caso concreto que examinaré, ciertamente de manera suscinta, es el de la filosofía de Descartes, de forma tal que dicha filosofía no será concebida al modo tradicional e internalista, o sea, tan sólo como otra respuesta más a problemas eternos de la filosofía, sino que intentaré mostrar que la filosofía cartesiana constituye una respuesta a problemas culturales extrafilosóficos. Ahora bien, pienso que la introducción de factores externos en la historia de la filosofía tiene por fin lograr los siguientes dos objetivos. Por una parte, sólo al resaltar el contexto cultural y socio-político especifico dentro del cual se afirma tal o cual tesis, podemos realmente determinar el significado histórico que tuvo dicha tesis.

Por otra parte, la introducción de factores externos nos permite comprender qué sentido cultural, y con ello social, tiene la filosofía, de tal manera que ella deja de ser concebida como un pensamiento que tiene por fin tan sólo resolver problemas intelectuales eternos, que precisamente por ello son irrelevantes a la vida real y a los cuales sólo pueden acceder unos cuantos dotados de "sensibilidad filosófica", y pasa a ser una actividad intelectual, ciertamente con un alto nivel de abstracción, que tiene por fin lidiar con (i.e. proponer, afirmar, justificar, criticar) valores culturales y sociales extrafilosóficos.

Considero que el propósito de la filosofía de Descartes es ofrecer una nueva visión de la realidad, o sea, una nueva visión de la naturaleza, del 
lugar que ocupa el hombre, y del creador de éstas, Dios, con el fin de darle prioridad intelectual y social a la ciencia moderna, o sea, con el fin de hacerle un lugar a la ciencia - que nunca antes tuvo- como una empresa cultural de suma importancia. Puesto en otros términos, el sentido extrafilosófico de la filosofía cartesina es el de proponer la sustitución de una cultura de tipo retórico-literaria (como es la escolástica y la humanista) por una cultura de tipo técnico científica ([11], p. 81; aún cuando esta caracterización se encuentra referida a Bacon, considero que también se aplica a Descartes).

Pasemos pues a ver algunos de los problemas que presentaba la ciencia moderna y cómo ciertas tesis filosóficas cartesianas acerca de la naturaleza y Dios constituyen una toma de posición frente a dichos problemas. Agruparemos estos problemas en internos y externos.

Los problemas internos fundamentales que aquejaban a la actividad científica entre 1590 y 1640 son los dos siguientes: a) La existencia de varias filosofías naturales en competencia y $b$ ) la poca claridad conceptual que existía en ese momento tanto respecto de una de las filosofias naturales, la mecanicista y matematizada, que Descartes apoya, como respecto de la investigación cientifica concreta fundada en dicha filosofía natural ([12], pp. 15-16, aunque el punto $(b)$ se encuentra desarrollado de otra manera). Ahora bien, dada esta situación en el ambiente "científico", el sentido que tiene la filosofía cartesiana es el de 1) argumentar que sólo una de las filosofías naturales en competencia, la mecanicista y matematizada, es la verdadera por estar bien fundamentada metafísicamente, 2) precisar, mediante un estudio metafísico, en qué consiste dicha filosofía natural, y 3) desarrollar una teoría física concreta, desarrollo que se encuentra posibilitado por ciertas tesis metafísicas. Trataremos ahora de desarrollar más estas ideas.

Por filosofía natural se entiende aquella parte de la filosofía que tiene por fin dar una visión general acerca de cómo es la naturaleza (i.e. qué entidades la conforman, qué estructura tiene la naturaleza y/o como interaccionan dichas entidades), y cómo es posible conocerla. Entre las filosofías naturales principales, que existían entre 1590 y 1640 , y que estaban en relativa competencia, están las siguientes. En primer lugar, la filosofía natural escolástica, o sea, la aristotélico-tomista, y que era la que principalmente se enseñaba en las universidades ([12], p. 25). Para esta filosofía, la naturaleza estaba compuesta por entidades cambiantes constituidas de materia y forma, de tal manera que conocer la naturaleza consistía en clasificar todos los tipos de cambio posible, y en explicarse el porqué de dichos cambios vía las cuatro causas aristotélicas. En segundo lugar, otra filosofia natural era el naturalismo, orientado hacia la magia, alquimia y astrología, y de inspiración neoplatónica y hermé- 
tica. Para esta filosofía, el supuesto fundamental es que la naturaleza está animada, o sea, que todos los entes que la conforman tienen vida y percepción. Entre los autores pertenecientes a esta corriente están, por ejemplo, Bruno, Gilbert y, parcialmente, Kepler. En tercer lugar, otra filosofía natural era el atomismo, cuyos exponentes fueron Bruno y, principalmente, Gassendi. Finalmente, en cuarto lugar, en la primera mitad del siglo xvir hay un fuerte movimiento hacia una filosofía natural mecanicista, en autores como Galileo, Kepler (parcialmente), Gassendi y Hobbes. ([12], pp. 24-33; [13], pp. 29-31).

Por mecanismo se entiende aquella filosofía natural según la cual la naturaleza está compuesta solo de materia y movimiento, y por lo cual, explicar cualquier fenómeno natural consiste en mostrar cómo éste es causado por partículas de materia, que tienen determinada forma geométrica, y con determinada cantidad de movimiento. En palabras de Descartes: "He descrito esta tierra y todo este mundo visible a manera de máquina, no considerando en él sino las formas y los movimientos [de sus partes]." ([6], Pr. IV, 188, p. 230). Destaquemos varias ideas que aparecen en esta cita: 1) el mecanicismo toma como modelo a la máquina, en el sentido de que se tratará de explicar a cualquier fenómeno natural de la misma manera en que se explica cualquier máquina, 2) así, el explicar un fenómeno natural consiste en explicar el mecanismo que lo causa, 3) el mecanismo se describe en términos solamente de partículas de materia, que tienen determinada forma geométrica y que pueden o no estar en movimiento, y 4) podemos notar que el mecanicismo es un pensamiento composicionalista (al igual que el atomismo), ya que se pretende explicar el todo (o sea, el fenómeno natural) mediante el examen de sus partes (o sea, las partículas materiales de que está compuesto).

Ahora bien, en Descartes encontramos que esta filosofía natural mecanicista está apoyada o fundada por su metafísica. En efecto, recordemos que de acuerdo con su tesis dualista, existen sólo dos tipos de sustancias creadas, el cuerpo, o sea, la materia, y el alma. El cuerpo o materia se caracteriza por tener el atributo de la extensión o tridimensionalidad, y por lo cual cualquier pedazo de materia sólo tiene por modos o propiedades cierta magnitud en largura, anchura y altura, cierta forma geométrica, y, o bien tiene cierta cantidad de movimiento, o está en reposo (p. ej. [5], Med. V, p. 179). Claramente, de su tesis metafísica dualista se sigue el mecanicismo, o sea, la idea de que la naturaleza sólo está compuesta de materia y movimiento.

En su filosofía natural Descartes no sólo sostuvo la tesis del mecanicismo sino que también sostuvo que el conocimiento de la naturaleza, i. e. la física, debía ser matematizado (por ejemplo [5], Med. V, p. 185). A esta última tesis también le dio un fundamento metafísico, ya que su tesis 
dualista y su definición de materia como extensión, implican que las únicas propiedades que puede tener la materia son todas ellas tratables matemáticamente. En efecto, las dimensiones en largo, ancho y alto son medibles, y la forma puede ser descrita geométricamente. El movimiento también es cuantificable ya que Descartes entiende por éste, sólo movimiento local, o sea, cambio de relaciones espaciales, de forma tal que la cantidad de movimiento que tenga un cuerpo se puede especificar estableciendo alguna función entre la distancia recorrida y el tiempo que se tomó en efectuar el recorrido. Recordemos que estas son las únicas propiedades que pueden tener los cuerpos; en verdad, los cuerpos no tienen olor, color, sabor, etc. ya que éstos no son modos de la extensión (p. ej. [5], Med. III, p. 164), y por otra parte, dada la tesis dualista resulta que los cuerpos son pura pasividad, o sea, no tienen ninguna propiedad o fuerza interna que cause que se muevan por sí mismos ([5], Med. II, p. 151), o sea, los cuerpos, de acuerdo con la metafísica cartesiana, no tienen propiedades que (al menos en principio) no están sujetas a un tratamiento matemático. Por otra parte, Descartes también dio una razón epistemológica por la cual la física debía matematizarse, sosteniendo que si la matematización se entendía como reducción de la física a la matemática, entonces la física se convertiría en un conocimiento tan cierto como lo es el de la matemática (carta a Mersenne, mayo 27, 1938; cit., en [4], p. 53).

Notemos que en la filosofía natural cartesiana aparecen unidas dos concepciones que no necesariamente van siempre unidas: el mecanicismo y la descripción matematizada de la realidad ([4], p. 53, [3] p. 1). Así, por ejemplo, en Galileo encontramos descripciones matematizadas de algunos fenómenos naturales como la caída libre y el movimiento pendular, pero no explicaciones mecanicistas de estos fenómenos. En cambio, y un tanto extrañamente, en la teoría física de Descartes encontramos explicaciones mecanicistas de fenómenos tales como el movimiento planetario o el magnetismo, pero que no están matematizadas. Por otra parte, estas concepciones no son incompatibles entre sí, ya que el mecanicismo es una tesis acerca de la causación física de los fenómenos, mientras que la tesis de la matematización especifica con qué lenguaje han de describirse los fenómenos. O sea, la tesis del mecanicismo y la de matematización son tesis independientes, que en Descartes aparecen conjuntamente.

Así pues, la filosofía cartesiana pretende mostrar que sólo una de las filosofías naturales en pugna a principios del siglo xvir es la correcta, ya que está bien fundamentada metafísicamente, mientras que las otras son incorrectas por presuponer una metafísica falsa. En efecto, la tesis del dualismo mente-cuerpo a la vez que sirve para fundamentar al mecanicismo matemático, también sirve para atacar al naturalismo ([27], p. 30; 
[13]), en tanto que implica que la concepción de una naturaleza animada, o sea, con propiedades espirituales es falsa: sólo la mente tiene un carácter espiritual, mientras que la naturaleza no lo tiene por ser pura materia pasiva, que si tiene movimiento, esto se debe a una causa externa (el impacto entre dos cuerpos). La tesis del dualismo igualmente sirve para derrumbar la filosofía natural aristotélica ([13], p. 31), porque al negar la validez de las distinciones filosóficas aristotélicas entre materia y forma, y esencia y accidentes (ya que la llamada "forma sustancial", o esencia de una sustancia, tiene un carácter animista por ser principio interno de actividad, mientras que el dualismo cartesiano le niega toda espiritualidad, y con ello actividad auto-causada a la naturaleza), se niega con ello a la doctrina de las cuatro causas, y a la filosofía natural aristotélica (con su distinción básica entre movimiento natural y violento, y la doctrina asociada de los lugares naturales), — que se apoyaba en la metafísica aristotélica.

Por otra parte, si bien había un movimiento general durante la primera mitad del siglo xvII a dar explicaciones mecanicistas y matematizadas de la naturaleza ([13], p. 30), el hecho es que no había una claridad conceptual respecto de éstas. Así, por ejemplo en Kepler conviven explicaciones mecanicistas y matematizadas del movimiento planetario con tesis naturalistas como de la existencia de un alma del mundo. $\mathrm{O}$ bien, en Galileo encontramos tanto descripciones matematizadas de fenómenos naturales como restos de la filosofía natural aristotélica. Ahora bien, lo que Descartes logra al remontarse a la filosofía es precisar conceptualmente exactamente en qué consiste la visión mecánico-matemática de la naturaleza y con ello qué otras concepciones de la naturaleza quedan excluidas.

Resumiendo, uno de los sentidos principales que tiene la filosofía cartesiana, y particularmente, la tesis dualista, es constituir una toma de posición frente al problema cultural de la existencia de diversas filosofías naturales, desarrollando exactamente en qué consiste la filosofía natural mecánico-matemática, y mostrando que sólo ésta es la correcta frente a las otras, por encontrarse bien fundamentada metafísicamente.

Ahora bien, vimos arriba que el mecanicismo privilegia como modelo a la máquina, en el sentido de que se sostiene que todo fenómeno ha de ser explicado de la misma manera en que se explica una máquina (i.e. vía la ciencia de la mecánica, que hasta finales del xvi la parte más desarrollada de ésta era la estática). Cabe preguntarse qué factores posibilitaron el surgimiento de la concepción mecanicista de la naturaleza. Parecería ser que el principal es el fuerte desarrollo del saber técnico en el siglo xvi. Veamos.

En el siglo xvr ocurrió un aumento notorio de la importancia econó- 
mica de las artes mecánicas, principalmente de la metalurgia, minería, navegación y artes militares ([11], p. 45). Esto tuvo las siguientes dos consecuencias. En primer lugar, el intento de resolver los problemas técnicos que planteaban el desarrollo de las artes mecánicas impulsó el desarrollo del saber técnico, o sea de un saber no meramente basado en hallazgos empíricos, sino también en las llamadas ciencias aplicadas ([1], p. 71; [7], p. 344), de las cuales la más avanzada era la mecánica ([1], p. 28). Es así que encontramos que la literatura del siglo xvi es rica en tratados de carácter técnico, y sobre todo en tratados sobre máquinas ([11], pp. 27, 50). En segundo lugar, el gran aumento de la importancia económica de Ias artes mecánicas contribuyó a una revaloración de éstas -revaloración que parece estar ligada también al ascenso de la burguesía y la consolidación de la monarquía ([11], p. 39). Esta revaloración se dio en diversos planos: a) se comenzó a revalorar el trabajo manual sobre todo el de los "ingenieros" como un trabajo digno ([11], p. 25), p. 25), b) a nivel de las instituciones educativas se dio una cierta tendencia a sustituir una educación puramente literaria por una preparación más técnica ([11], p. 22), y c) el saber técnico asociado a las artes mecánicas comenzó a valorarse como un saber valioso, como una forma de conocimiento (revaloración que aparece en autores tales como Bacon, Vives, Palissy, Rabelais, Vesalis) ([11], pp. 17-20). Ahora bien, la valoración del saber técnico como un conocimiento, hizo posible la compenetración entre la ciencia y la técnica ([11], p. 40; [12], p. 4), que es una característica peculiar a la ciencia moderna. Esta compenetración se dio en diversos niveles: a) la ciencia tomó de la técnica ciertos valores acerca del sentido que tiene hacer ciencia, ciertas características generales que debe tener la teoría científica y el modo en que se ha de desarrollar la ciencia (cfr. más adelante); b) en la ciencia moderna se comenzó a utilizar de manera notoria instrumentos científicos, lo cual fue posible gracias a la existencia de un saber técnico (p. ej. en óptica); c) ciertos problemas importantes al saber técnico de aquella época influyeron en el desarrollo teórico científico - p. ej. problemas en balística y en la construcción de relojes precisos (importante para la navegación) fueron tratados sistemáticamente por Galileo, Huygens, etc., d) el saber técnico, y principalmente la mecánica, fue tomado como modelo por la filosofía natural mecanicista. Así pues, parecería ser que uno de los factores importantes que permiten explicar el desarrollo y aceptación de la filosofía mecanicista en el siglo xvi es la existencia de un saber técnico en pleno desarrollo, sobre todo en el campo de la mecánica (aplicada), y el hecho de que el saber técnico comenzó a valorarse como una forma de conocimiento de la naturaleza.

Es interesante que la utilización de la máquina como modelo por la 
filosofía mecanicista rompe con la tradición aristotélica y medieval, para la cual hay una distinción ontológica entre las cosas naturales (cuyo principio de actividad característica es la esencia) y las cosas artificiales (que carecen de dicho principio), mientras que para la filosofía mecanicista hay una identidad entre el producto del "arte" o la técnica y las cosas naturales ([4], p. 53; [11], pp. 52 y 131). En palabras de Descartes:

Y para esto no me fueron de escasa ayuda las máquinas; en efecto, no hago ningún distingo entre ellas y los objetos naturales, sino en que las operaciones de las máquinas se realizan con instrumentos tan grandes que se perciben fácilmente por el sentido, pues eso se requiere para que puedan ser fabricados por los hombres. Pero en cambio, los electos naturales dependen casi siempre de órganos tan pequeños que escapan a todo sentido. $Y$ en verdad no hay casi ninguna proposición en la Mecánica que no se extienda también a la Física, de la que es una parte o especie; ni le es menos natural al reloj, compuesto de éstas o esas otras ruedas, indicar las horas, que al árbol, nacido de tal o cual semilla, producir frutos tales. Por lo cual, así como los que están ejercitados en considerar las máquinas automáticas, cuando ven el uso de máquina y miran sus partes, conjeturan fácilmente por ellas como están hechas otras que no ven; así también he intentado investigar por los efectos sensibles y las partes de los cuerpos naturales, cuales son sus causas y las partículas imperceptibles ([6], Pr. IV, 203; p. 240).

Essta identificación de los objetos naturales con los productos del arte, y la consideración de que los productos del arte -básicamente, las máquinas- son la clave para conocer los mecanismos (imperceptibles) que causan los fenómenos naturales, posibilita el que el saber técnico y el científico puedan relacionarse - ya sea que el saber técnico retome los conocimiento adquiridos por la ciencia o viceversa.

Con respecto al ideal de matematización del conocimiento de la naturaleza, podemos decir que éste ya gozaba de una cierta aceptación en la segunda mitad del siglo xvi, dada la vigencia del platonismo en un clima de ataque el escolasticismo, y dada la existencia de una serie de ciencias matematizadas más o menos bien desarrolladas, como la astronomía, la optica y la estática ([12], p. 14).

Sostuvimos arriba que la filosofía cartesiana no sólo tiene como uno de sus objetivos el de mostrar que la filosofía natural mecánico-matemática es la única correcta frente a las otras por estar fundamentada metafísicamente, sino que también otro de sus objetivos es el de servir de base para la construcción de una teoría física concreta, que obviamente deberá ser mecanicista y matematizada - aunque, de hecho, Descartes no 
logró desarrollar una física plenamente matematizada. Examinemos brevemente esta segunda afirmación.

La física cartesiana tiene los dos siguientes principios: 1) El principio de inercia, según el cual todo cuerpo en estado de reposo o de movimiento uniforme y rectilíneo, se conserva en dicho estado a menos que una causa externa actúe sobre él ([6], $P r$. II, 37 y 38, pp. 54-5). 2) El principio de colisión, según el cual la única causa externa que puede alterar el estado de reposo o movimiento uniforme rectilíneo es la colisión de ese cuerpo y otro (s), donde la colisión es tal que la suma de la cantidad de movimiento (i.e. el producto del tamaño por la velocidad escalar del cuerpo) de todos los cuerpos que participan en la colisión se conserva (i. e. es la misma antes, que después, de la colisión) ([16], Pr. If, 40, p. 56).

Como es claro, estos dos principios de la física cartesiana son principios de conservación del movimiento, y por ello Descartes los fundamenta en la tesis filosófica de la inmutabilidad de Dios ([16], Pr. II, 36, p. 53). Otras tesis filosóficas en que se fundamentan, como diría Descartes, de las que se "derivan" dichos principios físicos, son las siguientes:

1) El principio de inercia, sobre todo con su afirmación de que el movimiento uniforme rectilíneo es un estado que se conserva por sí mismo sin requerir de ninguna causa o fuerza, se fundamenta, en el sentido de que resulta pensable o aceptable, en las siguientes dos tesis filosóficas. a) La materia es pasiva, y por tanto, carece de fuerza propia para causar $\mathrm{u}$ oponerse al movimiento, y por lo cual un cierto tipo de movimiento puede ser un estado. b) La tesis de que el movimiento es sólo cambio de relaciones espaciales, y no un proceso como sostuvo Aristóteles, permite pensar que un cierto tipo de movimiento es un estado.

2) El principio de la colisión está fundamentado también en la tesis filosófica de que la materia es idéntica a la extensión o espacio de donde se sigue que no hay espacios vacíos ([6], $\operatorname{Pr}$. II, 16, p. 44) y, por tanto, que no es posible la acción a distancia, de tal forma que toda interacción entre pedazos de materia sólo puede darse vía la colisión o choque.

Así pues, hemos visto cómo se cumple el ideal deductivo del conocimiento de Descartes: la filosofía (básicamente, la tesis dualista) fundamenta o justifica una determinada filosofía natural y una teoría física concreta. Esto nos revela que la filosofía en Descartes aparece como un pensamiento muy abstracto que tiene por objetivo no precisamente el de responder a problemas intelectuales perennes de la humanidad, sino como un instrumento que permite resolver problemas concretos culturales, que en el caso de Descartes eran, principalmente, el de la construcción y justificación de una nueva ciencia.

Ahora bien, la construcción de esta nueva ciencia, no sólo se enfren- 
taba a los problemas "internos" de oponerse a otras filosofías naturales vigentes en ese momento y de requerir de una precisión conceptual de su aparato teórico, problemas a los que Descartes responde filosóficamente ofreciendo una concepción, mecánico-matemática, de la naturaleza, sino que también se enfrentaba a problemas "externos". Estos problemas externos son básicamente dos: a) la existencia de un clima de escepticismo bastante generalizado desde finales del xvi, y $b$ ) un conflicto abierto con la institución religiosa, principalmente de orden cristiano. Examinemos brevemente cada uno de estos puntos y cómo Descartes los enfrenta filosóficamente.

Desde 1560 Europa comenzó a entrar en una etapa de crisis general que se acentuó durante la primera mitad del siglo xvir. Esta crisis fue tanto económica (hubo un desplome de la economía), como políticoreligiosa-social (i.e. levantamientos populares, guerras religioso-políticas), y como espiritual (caracterizada por la existencia de muy diversas corrientes de pensamiento, por una actitud de pesimismo, y por epidemias de satanismo ([3], pp. 119-136; [12], p. 35). Esta crisis espiritual puede definirse también como una crisis general de autoridad, o sea, como la perdida de tradiciones bien establecidas, tanto a nivel intelectual (donde coexisten el neo-escolasticismo, el humanismo y la filosofía moderna) como a nivel religioso (cuya expresión más obvia es la Reforma y la Contrarreforma). Dada esta crisis de autoridad, el pensamiento escéptico (con representantes como Sánchez, Montaigne, Charrón) tuvo gran auge, sobre todo durante las décadas de los veinte y treinta del siglo xvir. Esta actitud escéptica no sólo se manifestó con respecto a la credulidad religiosa, sino también con respecto a la posibilidad del conocimiento ([2], p. 34; [4], p. 16).

Otro de los objetivos fundamentales de la filosofía cartesiana fue, precisamente, la de combatir al pensamiento escéptico vigente en su época. Esto lo hizo Descartes de las siguientes dos formas. En primer lugar, sostuvo que el conocimiento, para ser tal, debería ser cierto. E intentó mostrar que sí era posible tener conocimiento cierto argumentando que 1) las ideas claras y distintas, y particularmente, las ideas innatas, son verdades ciertas, y 2) que la ciencia (i.e. la física) es cierta por estar fundada en, o ser deducible de, verdades metafísicas ciertas (como el dualismo) (cf. carta a Mersenne, mayo 27, 1638; cit., en [4], p. 53). En segundo lugar, Descartes también intentó atacar al escepticismo religioso sostenido que tenemos un conocimiento cierto de la existencia de Dios, y que, además, Dios es la garantía de todo otro conocimiento cierto que podamos tener.

Por otra parte, otro problema fundamental al cual se enfrentaba la nueva ciencia era la oposición a ésta por parte de la Iglesia cristiana. 
Si bien en cada caso concreto de ataque a pensadores modernos, intervinieron una serie de factores particulares, en términos generales parecería ser que la razón principal por la cual la Iglesia llegó a atacar a la ciencia moderna era por el hecho de que varios pensadores - notablemente Bruno y Campanella- habían unido doctrinas pertenecientes a la nueva ciencia con doctrinas claramente anticristianas ([9], p. 80; [12], p. 28). Así, por ejemplo, en el pensamiento de Bruno se encuentran asociados el copernicanismo, el atomismo, la alabanza a las matemáticas, con un programa de reforma religiosa en el que se sostiene que la religión cristiana debía ser sustituida por la religión hermética originaria. Frente a este estado de cosas, los defensores de la nueva ciencia sostuvieron, o bien, que la ciencia y la religión no deben entrar en conflicto porque sus doctrinas pertenecen a distintos ámbitos, o bien, que la ciencia y la religión no tienen porqué entrar en conflicto dado que se apoyan mutuamente. Descartes optó por esta segunda alternativa, haciendo a Dios un elemento central de su pensamiento filosófico. En éfecto, por una parte, sostuvo que Dios era la garantía de todo conocimiento cierto, y por la otra fundó los principios de su física en la inmutabilidad de Dios. Es importante notar que, aún cuando Descartes hizo de Dios un elemento central en su pensamiento filosófico, su pensamiento no es teocéntrico, como el medieval, dado que se concibe a Dios de tal forma que sirve a los objetivos del sistema filosófico, ([2], p. 56) y no viceversa - así, por ejemplo, la existencia de Dios, y la determinación de sus propiedades cardinales, dejan de ser cuestión de dogma y pasan a ser cuestiones establecidas por la razón ([10], pp. 94-95). Y es precisamente por ello, por haber socabado las bases de la autoridad religiosa, por lo que la obra cartesiana fue condenada por Roma en 1663 ([4], p. 24).

Hemos visto así algunos de los problemas culturales frente a los cuales la filosofía cartesiana constituye una respuesta. Más concretamente, su concepto filosófico de naturaleza como materia y movimiento cuantificables intenta tanto aclarar conceptualmente los fundamentos de la nueva física, como atacar a otras filosofías naturales; y su uso del concepto de Dios intenta mostrar que la nueva ciencia y la religión católica son tales que se apoyan mutuamente. Resta, pues, por ver que lugar ocupa el hombre según la filosofía cartesiana.

Parecería ser que para la filosofía cartesiana el valor más importante que ha de perseguir el hombre es el conocimiento, y esto porque el conocimiento (de la naturaleza) le permitirá transformar a la naturaleza para mejorar su condición (material) -ideal que se opone a la concepción de Aristóteles, del escolasticismo y ciertas versiones del humanismo, en las que el énfasis es en el mejoramiento espiritual del hombre. Es por ello que en la filosofía moderna en general, la epistemología pasa a ocu- 
par un lugar preponderante; aunque también parece haber influido en esta revalorización de la epistemología el intento de resolver en el plano filosófico la crisis escéptica de la época, y particularmente, las crisis en la filosofía natural y en las teorías cientificas que anteriormente mencionamos.

Veamos con más detalle en qué consiste la visión moderna del conocimiento.

Con respecto a cuál es la finalidad (extracientífica) que tiene la ciencia, Descartes la enuncia de manera muy clara:

Pero tan pronto como hube adquirido algunas nociones generales respecto de la Física... creí que no podía guardarlas escondidas sin pecar grandemente en contra de la ley que nos obliga a procurar, en la medida en que nos sea posible, el bien general de toda la humanidad. Pues ella [i.e. las nociones de Física] me hicieron ver que es posible obtener conocimiento que es muy útil en la vida, y que, en vez de la filosofía especulativa que se enseña en las Escuelas, podemos encontrar una filosofía práctica por medio de la cual, conociendo la fuerza y la acción del fuego, agua, aire, las estrellas, los cielos y todos los otros cuerpos que nos rodean, tan distintamente como conocemos las distintas técnicas [crafts] de nuestros artesanos, podemos de la misma manera [que las distintas técnicas] utilizarlos para todos aquellos usos para los que están adaptados, y así convertirnos en los dueños y poseedores de la naturaleza. Esto no es deseable meramente con vistas a la invención de una infinidad de artes y técnicas que nos permita distrutar sin ningún problema de los frutos de la tierra y de todas las cosas buenas que se encuentran alli, pero también principalmente porque trae consigo la preservación de la salud ([5], D. M. VI, pp. 119-20).

Así, para Descartes (al igual que para Bacon y muchos otros pensadores modernos) la ciencia mecanicista tiene por fin permitirle al hombre dominar el mundo, i.e. actuar sobre el mundo tanto para obtener una mejora de las condiciones materiales de la vida humana (cfr. también [5], D.M. I, p. 84) como para conservar la salud. O sea, el concepto moderno de ciencia establece una cierta identificación entre el conocer y el hacer o construir: lo que verdaderamente se puede conocer es lo artificial (la máquina), lo construido y reconstruible ([11], p. 137). Es muy importante notar que este ideal de dominio del mundo es el ideal u objetivo de la técnica, de tal forma que al poner en relación a la ciencia mecanicista con la técnica, Descartes no sólo toma de la técnica ciertos modelos para la ciencia (como vimos anteriormeńte), sino que también toma de la técnica la finalidad que debe tener la ciencia. Por 
otra parte, como se señala en el texto citado, la ciencia mecanicista puede tener por objetivo la dominación del mundo precisamente por su relación con la técnica: en la medida en que la ciencia mecanicista tiene por modelo a la máquina, ésta puede ofrecer guías para el desarrollo de la técnica. Esta nueva concepción del conocimiento, como un cuerpo de ideas que debe tener un fin práctico y no meramente especulativo, no sólo tiene su origen en el que la ciencia se haya puesto en relación (por lo menos teóricamente ([7], p. vi)) con la técnica, sino que también proviene del pensamiento renacentista, principalmente del naturalismo, con su concepto del hombre-mago ([8], p. 47; [12], pp. 29-30).

Los pensadores modernos también tomaron de la técnica ciertas características que debería tener el conocimiento científico. La principal característica en que centraron su atención fue en la idea de progreso: consideraron que, al igual que el conocimiento técnico, el conocimiento científico debería poder progresar, y que esto sería posible en la medida en que muchos pensadores distintos colaboran en la gradual construcción de las teorías científicas ([11], p. 67; [12], p. 14; [15], pp. 251-3). En palabras de Descartes:

...comunicar fielmente al público lo poco que he descubierto, y rogar a todas las personas de buena voluntad a ir más allá contribuyendo, cada una de acuerdo a sus propias inclinaciones y habilidades, a los experimentos que deben realizarse, y después a comunicar al público todas las cosas que pudieran haber descubierto, con el fin de que el último pueda comenzar donde los anteriores se quedaron, y así, juntando las vidas y los esfuerzos de muchos, podamos colectivamente ir más allá de lo que cualquier persona en particular lograría ([5], D.M. VI, p. 120).

Esta idea de progreso científico se opone a la concepción clásica y medieval de la actividad científica. En efecto, para la concepción mo derna el conocimiento científico se desarrolla gradualmente (cfr. [5], $D . M$. I, p. 82), es un lento construir nunca acabado, y tal que este desarrollo se debe a la colaboración entre muchos individuos, ideas que implican que el criterio de verdad o justificación ya no es mas el principio de autoridad. En cambio, para la concepción clásica y medieval, el conocimiento se obtiene por los logros de unos pocos individuos eminentes, que pasan a ser considerados como autoridades, y donde la actividad cognoscitiva (o científica) consiste básicamente en la aclaración a través de comentarios de las obras de las autoridades, y en la transmisión de este saber ya dado. Ahora bien, este ideal moderno de progreso científico no fue meramente un ideal, sino que logró materializarse en los siglos xvir y xviII. En efecto, por una parte, para que fuese posible 
el trabajo científico cooperativo se requería de la comunicación entre los pensadores de los logros obtenidos; es por ello que en el siglo Xvir surgieron una gran cantidad de centros de reunión y academias que posteriormente dieron lugar a instituciones científicas - principalmente la Royal Society y la Academie Royale des Sciences-; también se encuentra una gran actividad epistolaria que posteriormente se institucionalizó con la creación de revistas científicas -como, por ejemplo-, la Transactions of the Royal Society. Por otra parte, el ideal de desarrollo científico acumulativo o progresivo vía un trabajo colaborativo se materializó en el hecho de que la actividad científica se convirtió por primera vez en una actividad organizada de investigación, o sea, se convirtió en la actividad sistemática de proponer problemas y resolverlos dentro de un esquema teórico dado, y que es lo que le permitió a la ciencia desarrollarse acumulativa y cooperativamente ([12], pp. 11, 13; [15], p. 253). En otros términos, el que la ciencia moderna fuese la primera en desarrollarse como "ciencia normal" (en el sentido kuhniano) se debe a que la ciencia moderna estaba animada por el ideal de progreso científico (i.e. desarrollo acumulativo y cooperativo) -aunque, por supuesto, también fue requisito para la "normalización" de la ciencia moderna el que se contara con un esquema teórico lo suficientemente bien desarrollado y rico que pudiese funcionar como guía o paradigma para la investigación sistemática, esquema que históricamente resultó ser el newtoniano.

Para concluir, tomando en consideración factores extrafilosóficos podemos ver que el sentido que tiene la filosofía cartesiana es abogar por la instauración de una cultura científico-técnica frente a la anterior cultura de tipo retórico-literaria. Para la nueva cultura científico-técnica el valor principal del hombre es el de desarrollar una ciencia que pueda progresar y que le permita el dominio del mundo natural. La única ciencia que tiene estas propiedades, según Descartes, es la mecanicista y matematizada, y tiene estas propiedades precisamente por la intima relación que guarda con la técnica. Ahora bien, la manera en que Descartes aboga por el desarrollo de esta nueva ciencia no fue sólo destacando su importancia social, o sea, el que es un conocimiento que permite una práctica, sino argumentando también que era la única ciencia verdadera por estar bien fundamentada metafísicamente, que era absolutamente cierta, y que además era compatible con las doctrinas cristianas vigenites en su época. 


\section{BIBLIOGRAFIA}

[1] Clark, George Science and Social Welfare in the Age of Newton. 2a ed. Oxford: at the Claredon Press. 1970.

[2] Collins, James. God in Modern Philosophy. Londres: Routledge and Kegan Paul. 1960.

[3] Corvosier, Andre. Historia Moderna. Barcelona: Editorial Labor. 1977.

[4] Crombie, A. C. "Descartes, René du Perron: Natural philosophy..." en Dictionary of Scientific Biography; Charles Coulston Gilliespie (ed.), vols. 3-4. Nueva York: Charles Scribner's Sons., 1981.

[5] Descartes, R. The Philosophical Works of Descartes; tr. por E. Haldane y G. R. T. Ross. Vol. I. Cambridge: at the University Press. 1968.

[6] Descartes, R. Principios de la filosofia. Buenos Aires: Editorial Losada. 1951.

[7] Ch. Singer, E. J. Holmyard, A. R. Hall, y T. I. William. A History of Technology, ed. Vol. III. Oxford: at the Claredon Press. 1979.

[8] Le Noir, Timothy. The Social and Intellectual Roots of Discovery in Seventeenth Century Mathematics. Indiana University Ph. D. 1974.

[9] Rattansi, P. M. "Science and Religion on the Seventeenth Century", en The Emergence of Science in Western Europe, ed. por M. Crosland. Nueva York: Science History Publications, 1976.

[10] Ricci, Clemente. "Descartes y el problema religioso" en Descartes. Homenaje en el Tercer Centenario del "Discurso del método". Vol. II. Buenos Aires: Universidad de Buenos Aires. 1937.

[11] Rossi, Paolo. Los filósofos y las máquinas, 1400-1700. Barcelona: Editorial Labor.

[12] Schuster, John Andrew. Descartes and the Scientific Revolution. 1618-1634. An Interpretation. Ph. D. Dissertation, Princeton University. 1977.

[13] Westfall, Richard. The Construction of Modern Science, Mechanisms and Mechanics. Cambridge University Press. 1977.

[14] Williams, Bernard. Descartes: The Project of Pure Inquiry. Sussex: The Harvester Press. 1978.

[15] Zilsel, Edgar. "The genesis of the concept of scientific progress", en Roots of Scientific Thought. A cultural perspective, ed. por Ph. P. Weiner y A. Noland. Nueva York: Basic Books Publishers. 1958. 